Romeo L. Villarta Jr., MD, MPH ${ }^{1}$

Maria Luz M. San Agustin, RN, MClinAudio²

'Department of Epidemiology and Biostatistics College of Public Health

University of the Philippines Manila

${ }^{2}$ Ear Unit

Philippine General Hospital

University of the Philippines Manila
Correspondence: Dr. Romeo L. Villarta Jr.

Department of Epidemiology and Biostatistics

College of Public Health

University of the Philippines Manila

625 Pedro Gil St., Ermita, Manila 1000

Philippines

Phone: (632) 5260784

Telefax:: (632) 5254239

Email: rvillartajr@upm.edu.ph

Reprints will not be available from the author.

The authors declared that this represents original material that is not being considered for publication elsewhere in full or in part, in print or electronic media; that the manuscript has been read and approved by all the authors, that the requirements for authorship have been met by each authors, and that each author believes that the manuscript represents honest work.

Disclosures: The authors signed disclosures that there are no financial or other (including personal) relationships, intellectual passion, political or religious beliefs, and institutional affiliations that might lead to a conflict of interest.

\section{Auditory Steady State Response (ASSR) Frequency-Specific Thresholds with Absent Auditory Brainstem Response (click-ABR) Test Results among Filipino Children}

\begin{abstract}
Objective: Determine the frequency-specific thresholds of auditory steady state response (ASSR) of Filipino children with absent auditory brainstem response (click-ABR) results.

Methods: This is a cross-sectional study analyzing the frequency-specific thresholds of auditory steady state response (ASSR) of Filipino children with absent auditory brainstem response (click-ABR) results. The study population comprised of 99 pediatric patients referred for hearing assessment using electrophysiologic techniques at the Ear Unit of the Philippine General Hospital. The subjects underwent hearing threshold evaluation using both evoked-potential techniques (click ABR and ASSR) within a one-month period from January 2009 to March 2014. The ASSR results of patients with absent click-ABR were collected and analyzed.
\end{abstract}

Results: There were 99 patients who underwent both ABR and ASSR. Of the 65 patients with absent ABR thresholds results, 13 patients had unilateral absent ABR while 52 had bilateral absent $A B R$ results. The data of hearing tests from the combined 117 ears with absent $A B R$ hearing tests were collected.

The proportion of children with ASSR thresholds with absent ABR per frequency were:

- $500 \mathrm{~Hz}-45 / 117(38.5 \%)$

- $1000 \mathrm{~Hz}-76 / 117(64.0 \%)$;

- $2000 \mathrm{~Hz}-63 / 117(53.8 \%) ;$ and

- $4000 \mathrm{~Hz}-41 / 117$ (35.0\%).

The proportion of children with ASSR thresholds with absent ABR per number of frequencies were:

- 4 frequencies - 19/117 (16.2\%);

- 3 frequencies - 32/117 (27.4\%);

- 2 frequencies - $22 / 117(18.8 \%)$; and

- 1 frequency - 44/117 (37.6\%)

Conclusion: In the absence of click- ABR response, ASSR may provide information about the levels of severe to profound hearing loss among children. The criteria of selection of candidates for intervention (hearing aids or cochlear implantation) should include results from hearing evaluation not only from behavioral and ABR thresholds but also from ASSR thresholds. This may ensure that exclusion of some children with severe and profound hearing loss who may benefit from the intervention will be minimized.

Keywords: profound sensorineural deafness, evoked response audiometry, hearing thresholds

With the implementation of universal newborn hearing screening in the world including the Philippines, more children will be identified at birth with hearing loss and subsequently evaluated for intervention. For young children, early diagnosis of hearing loss and early intervention with amplification or cochlear implantation allow access to sound and the potential to develop speech, language and listening skills needed for oral communication. ${ }^{1,2}$ However, for a subset of hearing-impaired children with severe to profound hearing loss, current evaluation for

Philipp J Otolaryngol Head Neck Surg 2014; 29 (2): 7-9

(c) Philippine Society of Otolaryngology - Head and Neck Surgery, Inc. 


\section{ORIGINAL ARTICLES}

fitting of hearing aids or cochlear implants presents a special problem. Estimates of profound, early-onset deafness are around 4-11 per 10,000 children in the United States. ${ }^{3}$ Current testing for children with severe to profound hearing using click-ABR (Auditory Brainstem Response) and behavioral test methods is limited by the inability to obtain frequencyspecific thresholds. ${ }^{4}$

An absent auditory brainstem response does not allow meaningful conclusions about the amount of residual hearing. The estimation of residual hearing is relevant to the selection of a right habilitation strategy particularly in children with severe or profound hearing loss where the loss is overestimated by ABR. ${ }^{5}$

However, Auditory Steady-State Response (ASSR), an evoked potential test, can accurately measure auditory sensitivity beyond the limits of other test methods. ${ }^{4}$ The ASSR system's primary advantage over the standard evoked potential test is the ability to differentiate between severe and profound hearing loss as well as distinguishing between levels of profound hearing losses, e.g. the difference between a $90 \mathrm{~dB}$ and a $110 \mathrm{~dB}$ hearing loss. This ability to differentiate is crucial in instances where a cochlear implant is being considered as well as accurately fitted amplification. Unlike ABR testing which does not differentiate the severe and profound levels, the ASSR evaluation in combination with the behavioral methods currently used will make earlier identification of hearing loss even more accurate and this is essential for the management of infants with severe to profound hearing loss. ${ }^{6,7}$

To the best of our knowledge, there are no published Philippine studies that quantify the ASSR frequency-specific thresholds of children with severe and profound hearing loss diagnosed with negative or absent response to click-ABR. This study would provide an estimate of the numbers of possible children with residual hearing who may be excluded from hearing intervention if the decision is based only on behavioral and ABR hearing thresholds.

The objective of this study was to determine the frequency-specific thresholds of auditory steady state response (ASSR) of Filipino children with absent auditory brainstem response (click-ABR) results.

\section{METHODS}

The cross-sectional study involved analysis of the frequency-specific thresholds of auditory steady state response (ASSR) of Filipino children with absent auditory brainstem response (click-ABR) results.

\section{Sample Population:}

Children referred for hearing assessment at the Ear Unit of the Philippine National Ear Institute and Philippine General Hospital because of the inability of conducted behavioral tests to provide reliable estimates of hearing sensitivity were included in the study. The children who participated in this study underwent hearing threshold evaluation using both evoked-potential techniques (click ABR and ASSR).

\section{Data Collection:}

Medical and audiologic records containing the ABR and ASSR results of children seen from January 2009 to March 2014 were reviewed. Only the records of children who underwent both $A B R$ and
ASSR testing within a 1-month interval period were included in the study. A written informed consent of the parents or legal guardian of each child was solicited. The University of the Philippines Manila Ethics Review Board approved the research protocol. All patients were tested using the standard Testing Protocol for ABR and ASSR of the Ear Unit of the Philippine General Hospital. ${ }^{8}$ If deemed necessary using standard clinical procedure, the patient was sedated using chloral hydrate. Audiologic testing made use of the Biologic ${ }^{\circledR}$ Master ${ }^{\circ}$ II Multiple Auditory Steady-State Evoked Response machine (Natus Medical Incorporated, San Carlos, CA, USA).

The first test performed was the click-ABR. The results were recorded using Biologic Navigator ${ }^{\circledR}$ evoked potential system. Surface electrodes were applied to the high forehead (active), the ipsilateral mastoid process (ground) and the contralateral mastoid process (reference). Electrode impedances never exceeded 3kohms. The click rarefaction polarity stimulus consisting of $100 \mu$ s pulses of a maximum of $95 \mathrm{~dB} \mathrm{nHL}$ at a rate of $13.3 / \mathrm{sec}$ and a filter of $30-1500 \mathrm{~Hz}$ bandwidth with an amplifier gain of 10,000 . Time window of $20 \mathrm{msec}$ were used to record the click-ABR. At each presentation level, a minimum of 1500 sweeps was averaged. A $10 \mathrm{~dB}$ increment or decrement was used to determine the threshold. Threshold was defined by visual inspection of the waveform displayed on the computer screen.

ASSR testing immediately followed while the patient was still asleep or sedated. Patients were tested at $10 \mathrm{~dB}$ above the previously determined $A B R$ thresholds when available. Increments of $10 \mathrm{~dB}$ and decrements of $10 \mathrm{~dB}$ were used depending on the required number of sweeps per frequency and threshold. Patients tested for thresholds of $80 \mathrm{~dB} \mathrm{HL}$ and above were tested mono-aurally, one frequency at a time.

\section{Data Analysis:}

The results were codified as shown in Tables 1 and 2 .

$A$ retrospective review of the records of patients with absent $A B R$ results was performed and the data inputted in an Excel ${ }^{\circ}$ spreadsheet (Microsoft Corporation, USA) containing among others, the hospital record number, name, age, ABR thresholds and ASSR thresholds (for right and left ear). Descriptive statistical data analysis was performed using Stata $11^{\circ}$ statistical software (StataCorp LP, Texas, USA).

\section{RESULTS}

There were 99 patients who underwent both ABR and ASSR within a 1-month period from January 2009 to March 2014. There were 47 males $(52.5 \%)$ and 52 females (47.5\%) with mean age of 3.6 years (SD=1.9 and range: 0.6 - 10).

Of the 99 patients, 65 patients had absent ABR thresholds results: 13 patients with unilaterally absent $A B R$ results and 52 with bilaterally absent $A B R$ results.

The data of hearing tests from the combined 117 ABR hearing tests were collected.

The proportion of children with ASSR frequency-specific thresholds with absent $A B R$ per frequency is shown in Table 3. More than half of the patients with absent click-ABR had ASSR thresholds in the $1000 \mathrm{~Hz}$ (64\%) and $2000 \mathrm{~Hz}$ (54\%) levels. More than a third of patients had ASSR 
Table 1. Coding manual for data entry for ABR Thresholds

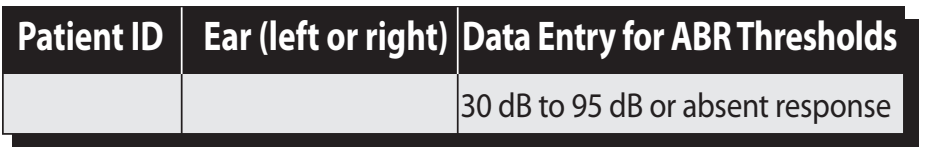

Table 2. Coding manual for data entry for ASSR Thresholds

\begin{tabular}{|l|l|l|l|}
$\begin{array}{c}\text { Patient } \\
\text { ID }\end{array}$ & $\begin{array}{c}\text { Ear } \\
\text { (left or right) }\end{array}$ & $\begin{array}{c}\text { ASSR THRESHOLDS } \\
\text { (Frequency) }\end{array}$ & \multicolumn{1}{|c|}{ Data Entry } \\
\hline & $500 \mathrm{~Hz}$ & $\begin{array}{l}20 \mathrm{~dB} \text { to } 114 \mathrm{~dB} \text { or } \\
\text { absent response }\end{array}$ \\
\hline & $1000 \mathrm{~Hz}$ & $\begin{array}{l}20 \mathrm{~dB} \text { to } 120 \mathrm{~dB} \text { or } \\
\text { absent response }\end{array}$ \\
\hline & $2000 \mathrm{~Hz}$ & $\begin{array}{l}20 \mathrm{~dB} \text { to } 120 \mathrm{~dB} \text { or } \\
\text { absent response }\end{array}$ \\
\hline & $4000 \mathrm{~Hz}$ & $\begin{array}{l}20 \mathrm{~dB} \text { to } 120 \mathrm{~dB} \text { or } \\
\text { absent response }\end{array}$ \\
\hline
\end{tabular}

Table 3. Proportion of children with ASSR Thresholds and absent click-ABR results:

\begin{tabular}{|c|c|}
\hline $\begin{array}{c}\text { ASSR THRESHOLDS } \\
\text { (Frequency) }\end{array}$ & Proportion \\
\hline $500 \mathrm{~Hz}$ & $45 / 117(38.5 \%)$ \\
\hline $1000 \mathrm{~Hz}$ & $76 / 117(64.0 \%)$ \\
\hline $2000 \mathrm{~Hz}$ & $63 / 117(53.8 \%)$ \\
\hline $4000 \mathrm{~Hz}$ & $41 / 117(35.0 \%)$ \\
\hline
\end{tabular}

Table 4. Proportion of children with multiple ASSR Thresholds frequencies and absent click-ABR results:

\begin{tabular}{|c|c|}
\hline $\begin{array}{c}\text { Number of ASSR } \\
\text { Thresholds frequencies }\end{array}$ & Proportion \\
\hline 4 & $19 / 117(16.2 \%)$ \\
\hline 3 & $32 / 117(27.4 \%)$ \\
\hline 2 & $22 / 117(18.8 \%)$ \\
\hline 1 & $44 / 117(37.6 \%)$ \\
\hline
\end{tabular}

thresholds in the $500 \mathrm{~Hz}(38 \%)$ and $5000 \mathrm{~Hz}$ (35\%) levels.

The proportion of children with ASSR frequency-specific thresholds with absent ABR per number of frequencies is shown in Table 4. Less than half (44\%) of the patients had ASSR thresholds in three or more frequencies. For those with other frequencies of ASSR thresholds, the results were still significant (1 frequency - 38\%; 2 frequencies - 19\%).

\section{DISCUSSION}

Relying on the evidence provided by click-ABR alone as basis for intervention planning for hearing loss such as prescription for hearing aid amplification and cochlear implantation would underestimate the number of children who may benefit from intervention. The results of the study show that around $35-64 \%$ of children with absent ABR results have residual hearing shown in the ASSR thresholds in each tested frequency. Furthermore, around $16 \%$ exhibit ASSR thresholds in 4 frequencies. Clearly these numbers are significant. These findings confirm the conclusions of several studies that in children with no response $A B R$, additional electrophysiologic testing be conducted to acquire a more complete assessment of the child's hearing. ${ }^{6,9}$

The study results also support the contention of Swanepoel and Hugo that preliminary results indicate that absent ABR and behavioral thresholds do not preclude the possibility of residual hearing, making the ASSR a primary source of information regarding profound levels of hearing loss. ${ }^{10}$

Health personnel involved in planning intervention for children with severe to profound hearing loss should consider that evidence based on behavioral and ABR hearing thresholds alone may exclude children with possible residual hearing. The benefit of the ASSR is that the results may provide more frequency-specific threshold information for children who have severe to profound hearing losses. This information would provide more precise data to proceed with hearing aid fittings or determining cochlear implant candidacy. It should be noted however that the Joint Committee on Infant Hearing $(\mathrm{JClH}) 2007$ Position Statement does not recommend ASSR as the sole measure of auditory status in newborn and infant populations."

Reliance on click-ABR alone for hearing evaluation may result in overestimation of the prevalence of hearing loss. Even in the absence of results of click- ABR thresholds, ASSR may provide information about the residual hearing of children with profound hearing loss. The criteria used in selection of candidates for intervention (hearing aids or cochlear implantation) should include results from hearing evaluation not only from behavioral and ABR thresholds but also from ASSR thresholds. This may minimize the exclusion of some children with profound hearing loss who may benefit from the intervention.

\section{REFERENCES}

1. Yoshinaga-Itano C, Sedey A, Coulter DK, Mehl AL. Language of early and later identified children with hearing loss. Pediatrics. 1998;102:1161-1171.

2. Moeller MP. Early intervention and language development in children who are deaf and hard of hearing. Pediatrics. 2000 Sep;106(3):E43.

3. Marazita ML, Ploughman LM, Rawlings B, Remington E, Arnos KS, Nance WE. Genetic epidemiological studies of early-onset deafness in the U.S. school-age population. Am J Med Genet. 993 Jun; 46(5): 486-491.

4. Stueve MP, O'Rourke CA. Comparison of auditory steady-state response, auditory brainstem response, and behavioral test method. Am J Audiol. 2003 Dec;12(2): 125-136.

5. Marttila TI, Karikoski JO. Comparison between audiometric and ABR thresholds in children. Contradictory findings. Eur Arch Otorhinolaryngol. 2006 May; 263(5): 399-403.

6. Firszt JB, Gaggl W, Runge-Samuelson CL, Burg LS, Wackym PA. Auditory sensitivity in children using the auditory steady-state response. Arch Otolaryngol Head Neck Surg. 2004 May; 130(5): 536-40.

7. Lee HS, Ahn JH, Chung JW, Yoon TH, Lee KS. Clinical comparison of the auditory steady-state response with the click auditory brainstem response in infants. Clin Exp Otorhinolaryngol. 2008 Dec; 1(4): 184-188.

8. Tan LC, Reyes-Quintos MR, Tantoco MC, Chiong CM. Comparative study of the auditory steadystate response (ASSR) and click auditory brainstem-evoked response (click ABR) Thresholds among Filipino Infants and Young Children. Philipp J Otolaryngol Head Neck Surg. 2009 Jan-Jun 24 (1): 9-12.

9. Kandogan T, Dalgic A. Reliability of auditory steady-state response (ASSR): comparing thresholds of auditory steady-state response (ASSR) with auditory brainstem response (ABR) in children with severe hearing loss. Indian J Otolaryngol Head Neck Surg. 2013 Dec; 65(Suppl 3):604-7.

10. Swanepoel D, Hugo R. Estimations of auditory sensitivity for young cochlear implant candidates using the ASSR: preliminary results. Int J Audiol 2004 Jul-Aug; 43(7): 377-382.

11. Joint Committee on Infant Hearing. Year 2007 position statement: Principles and guidelines for early hearing detection and intervention programs. Pediatrics. 2007 Oct; 120(4): 898-921. 